\title{
A first attempt to Intensity Interferometry with Iqueye
}

\section{Capraro ${ }^{1}$}

Department of Astronomy, University of Padova, Italy vic. Osservatorio, 335122 Padova, Italy

E-mail: ivan.capraro@dei.unipd.it

\section{G. Naletto ${ }^{2,3}$, C. Barbieri ${ }^{4}$, T. Occhipinti ${ }^{4}$, E. Verroi ${ }^{2,4}$, P. Zoccarato ${ }^{4,5}$ and A. Di Paola ${ }^{6}$}

Intensity Interferometry is a technique for the calculation of stars angular diameters that could in principle overcome some major disadvantages of traditional amplitude interferometry. This technique was first applied in 1956 by Hanbury-Brown and Twiss (HBT) in Narrabri (Australia), when they measured the diameter of several bright blue stars. Since then, the HBT technique nearly disappeared from astrophysics. Nowadays theory and technology are mature enough to guarantee a renewed exploitation of this effect in astronomy. Our group in Padua has built Iqueye, a high speed astronomical photon counting device, first tested at the ESO $3.5 \mathrm{~m}$ New Technology Telescope (La Silla, Chile) in January 2009. With the collected data, a posteriori we perform the calculation of $\mathrm{g} 2(\mathrm{x}, 0)$ as a first step to verify the zero-baseline correlation coefficient in a HBT intensity interferometer. In a similar way, g2 $(0, t)$ could be calculated. To perform such calculations, a dedicated software correlator has been designed, implemented and optimized and will be described in depth in the present paper. Although the size of the NTT does not allow an acceptable signal to noise ratio for calculating second order effects, our experiments demonstrate the value of an Iqueye-like instrument for HBT like experiments. Hopefully an improved version of IQuEye applied to larger telescopes, like the four $8 \mathrm{~m}$ VLTs and even to the future $42 \mathrm{~m}$ Extremely Large Telescope will be the perfect instrument for a novel exploitation of the photon stream from celestial objects.

Quantum of Quasars workshop

Grenoble, France

December 2-4, 200

\author{
Speaker \\ Department of Information Engineering, Univ. of Padova, Via Gradenigo, 6/A, I-35131 Padova, Italy \\ CNR/INFM/LUXOR, Padova, Italy \\ Department of Astronomy, University of Padova, Italy vic. Osservatorio, 335122 Padova, Italy \\ Interdepartmental Center of Studies and Activities for Space "G. Colombo", Univ. of Padova,Italy \\ INAF Astronomical Observatory of Roma, Italy
}




\section{Introduction}

We have build an instrument called IQuEye, capable of time tagging every photon coming from celestial sources with a time accuracy of 500ps over hours of acquisition. Considering the extreme capabilities of the instrument, it has been possible to perform a first attempt of quantum like analysis such as Correlation Spectroscopy[1] or a renewal of Habury Brown \& Twiss Interferometry[2]. IQuEye has been mounted on the ESO NTT Telescope in Chile and some targets have been acquired with the aim to perform HBT. This paper resumes some early results even though this kind of analysis would require a much larger collector with respect to NTT.

\section{The Instrument: IQuEye}

Iqueye (fully described in [5]) represents the second step of a program which started in 2005. At that time we proposed QuantEYE [3] as a possible focal plane instrument for OWL[4]. Iqueye's optical head is equipped with four Single Photon Avalanche Diode (SPAD) each one optically coupled with one fourth of the telescope pupil by means of a pyramid placed on the focal plane of a built in focal reducer. The four signals coming from the SPADs are collected and time tagged by a time-to-digital converter with a nominal resolution of 24 picoseconds rms. The optical design is represented in Figure 1. The time data are then sent to a storage device. A Rubidium oscillator, drift corrected through the PPS signal of a GPS receiver, references the time tags to UTC. The overall time accuracy is better than 500ps rms for each photon tag, during the whole observation. The maximum count rate that the system could handle in January 2009 was limited to $8 \mathrm{Mcps}$. A typical observing run of 60 min produces about 100 GBytes of time tags, each 28 bit long.

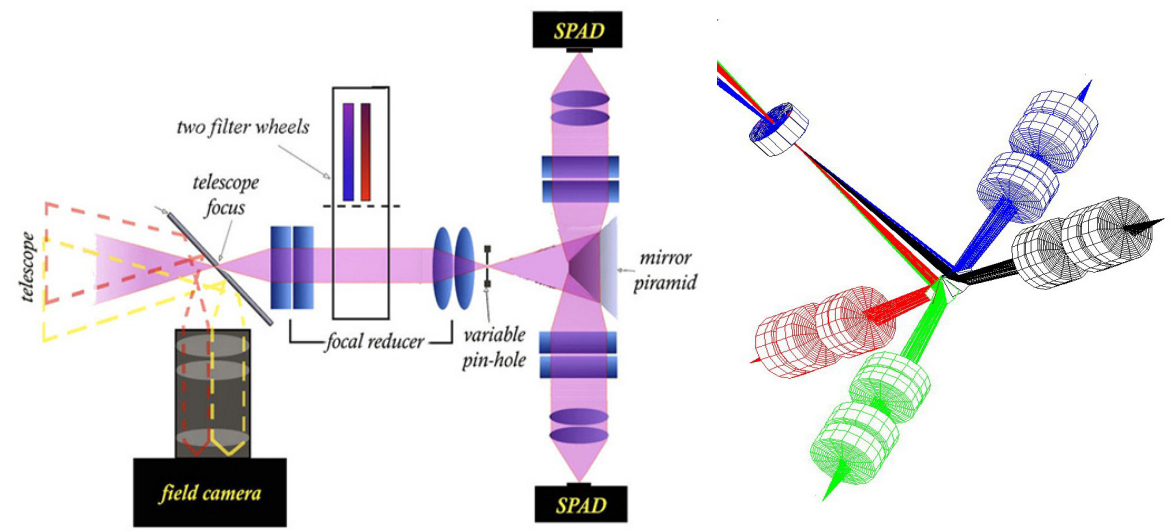

Figure 1: Optical Design of IQuEye. Left: the whole optical path from the telescope focal plane. Right: a particolar of the pyramid that splits the telescope pupil plane.

With this instrument used as photometer we already obtained several appreciable results described in [5] such as a very precise analysis of the crab pulsar light curve. 


\section{Quantum Astronomy with IquEye}

HBT intensity interferometry is performer by calculating the second order correlation of the intensity data $I(\boldsymbol{r}, \boldsymbol{t})$ by the following formula:

$$
g^{(2)}(\boldsymbol{d}, \boldsymbol{\tau})=\frac{\left\langle I\left(\boldsymbol{r}_{1}, t_{1}\right) I\left(\boldsymbol{r}_{2}, t_{2}\right)\right\rangle}{\left\langle I\left(\boldsymbol{r}_{1}, t_{1}\right)\right\rangle\left\langle I\left(\boldsymbol{r}_{2}, t_{2}\right)\right\rangle}
$$

where $\boldsymbol{d}=\boldsymbol{r}_{2}-\boldsymbol{r}_{1}$ is the baseline between two collectors and $\tau=t_{2}-t_{1}$ is the time-shift between photons. In particular HBT considers $\boldsymbol{\tau}=0$ and $\boldsymbol{d} \neq 0$ whereas when $\tau \neq 0$ and $\boldsymbol{d}=0$ correlation spectroscopy is performed. In both cases, when considering a photon counting device such as IQuEye the signal to noise ratio is given by :

$$
(S / N)_{R M S}=\frac{1}{2}\left(N_{1} N_{2}\right)^{1 / 2} \tau_{0}|\gamma(\boldsymbol{d}, \boldsymbol{\tau})|^{2}\left(T_{0} / 2 \Delta t\right)^{1 / 2}
$$

where $N_{l}$ and $N_{2}$ are the number of collected photons in the two collectors respectively, $\tau_{0}$ is the coherence time of the radiation, $\gamma$ is the complex degree of coherence, $T_{0}$ the exposure time and $\Delta \mathrm{t}$ is the coincidence windows used to perform the measurement [7].

The following figure reports a simulation of the SNR on an IQuEye like instrument attached to several telescopes. As one can see the SNR with a $4 \mathrm{~m}$ class telescope with the pupil divided by four such our case (blue in Figure) is more or less ten time better than the original Narrabry experiment but unfortunately the data stream for magnitudes with good SNR was not handled by our electronics. The other thing to notice is that the curves saturate at high fluxes due to detectors dead time.

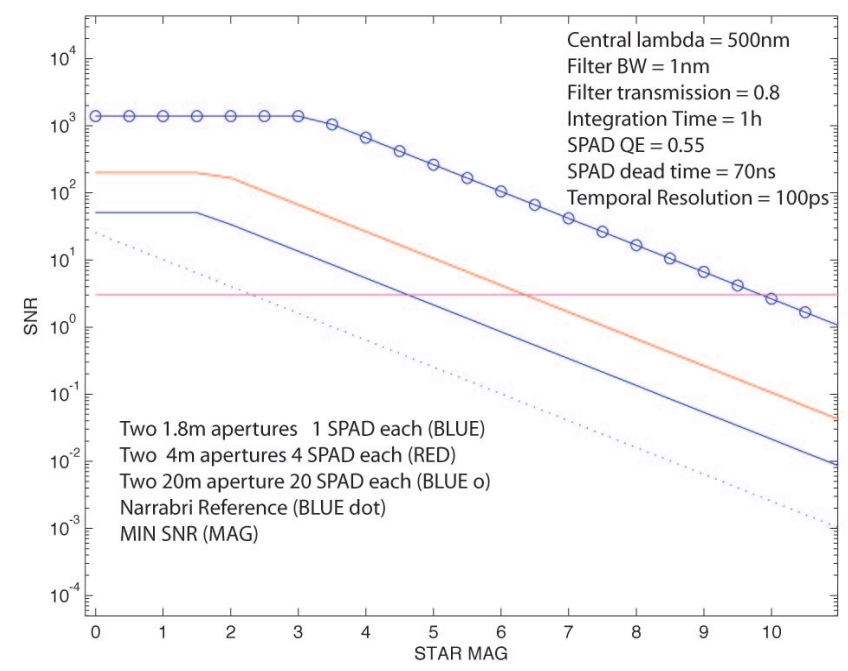

Figure 2: SNR of an IQuEye like instrument attached to different telescopes 


\section{The Software Correlator}

The philosophy of IQuEye is to store the time tag of every photon coming from the target, in this way a huge amount of data is stored, namely for example 100GB of data for one hour of observation. In order to handle the correlation of such data an ad hoc software correlator has been implemented. The software perform the time correlation directly working on the time tags $t_{i}$ using a different approach with respect to standard calculation. Normally the two sets of data are translated one respect to the other and at each translation the coincidences are counted whereas in this case first we calculate the differences in time between one time tag and all the others and then move forward to the next time tag, at every step suppose that the two photons where collected at times $t_{N}$ and $t^{\prime}{ }_{M}, t^{\prime}{ }_{M}>t_{N}$ we increase the correlation $C\left(t^{\prime}{ }_{M}{ }^{-} t_{N}\right)$ by one. This approach has several advantages with respect to other approaches namely i) is works on a smaller data set since it uses time tags and it does not require a binned intensity string; ii) it is faster because although the computational complexity is $\mathrm{O}\left(\mathrm{n}^{2}\right)$ working on a smaller data set is a big advantage, notice also that the application of standard FFT based approach is very hard with huge amounts of data; iii) it is more reconfigurable with respect to hardware correlators, it is possible to change the time bin, the number of files processed, pre process data ecc. The following figure illustrates the algorithm.
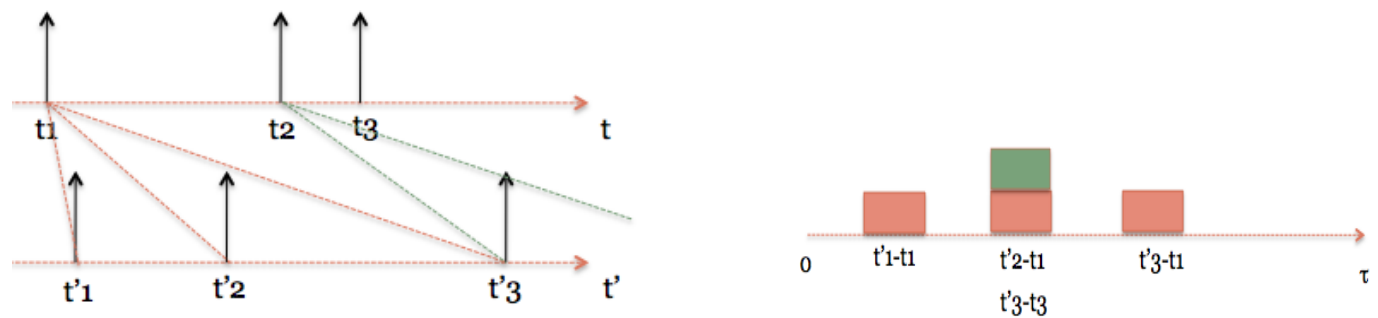

Figure 3: The software correlator works directly on the photon time tags building the correlation step by step. Left: the two photon streams. Rigth: the correlation.

\section{First Results}

We tried to perform zero baseline HBT on $\zeta$ Ori (HD 37743, V = 1.8, SpT $=09.5 \mathrm{Ib}$, with emission lines). HBT quoted a zero baseline correlation coefficient $g^{(2)}(0)-1=0.60 \pm 0.06$, very far from the value of 1 expected from a single unresolved star. Their explanation was that the difference is due to the resolution of the stellar disk, corresponding to an angular extent of the order of 0.5 mas for a uniformly illuminated disk. Actually, it is well known [6] that the $\zeta$ Ori is a triple star, with the secondary ( $\zeta$ Ori $\mathrm{B}, \mathrm{V}=4.20, \mathrm{SpT}=\mathrm{B} 0 \mathrm{III}$, flux $\mathrm{B} / \mathrm{A} \approx 0.1$ ) distant approximately $2 " .5$ to the South East, and therefore just at the edge of the used pinhole that defines the instrument filed of view. The tertiary weaker component is too far to be of concern in our data so with our setup we expect then $g^{(2)}(0)-1$ to be nearly one. Unfortunately we had to put a ND (Neutral Density) filter in our setup because we could not handle the data stream with our electronics. The following table resumes the results, to summarize we had four apertures, each is a quarter of the NTT pupil so there are two effective distances between the sub-pupil pairs. Here the resolving angle is $\theta \approx \lambda / \mathrm{d}$ with $\lambda=430 \mathrm{~nm}$. 
Table 1: Results for the calculation of zero baseline $\mathrm{g}^{(2)}(0)$ for $\zeta$ Ori.

\begin{tabular}{ccccc}
\hline \hline $\begin{array}{c}\text { Channel pair } \\
\text { (channel numbers) }\end{array}$ & $\begin{array}{c}\text { Effective distance } \\
d(\mathrm{~m})\end{array}$ & $\begin{array}{c}\text { Resolving angle } \\
\theta(\operatorname{arcsec})\end{array}$ & $\left\langle g^{(2)}(d)\right\rangle$ & $\begin{array}{c}\text { Standard } \\
\text { deviation }\end{array}$ \\
\hline A-B & 1.7 & 0.052 & 0.886 & 0.002 \\
A-C & 2.4 & 0.037 & 0.912 & 0.003 \\
A-D & 1.7 & 0.052 & 0.931 & 0.001 \\
B-C & 1.7 & 0.052 & 0.909 & 0.002 \\
B-D & 2.4 & 0.037 & 0.909 & 0.004 \\
C-D & 1.7 & 0.052 & 0.932 & 0.004 \\
\hline
\end{tabular}

Notice that NTT uses a de-rotator to keep the field of view with the same orientation for the whole observation, this implies that the baselines, projected onto the sky are different from each other even though the telescope's symmetry.

\section{Comments and Conclusions}

IQuEye is an instrument that can be used to perform quantum like measurement such as HBT. This paper showed some early results affected mainly by hardware limitations, nevertheless the method is indeed valid and valuable of further explorations. An improved version of IQuEye is at the NTT right now (December 2009), and further observations will be attempted.

\section{References}

[1] Dainis Dravins and Claudio Germana, Photon Correlation Spectroscopy for Observing Natural Lasers, in proceedings of High Time Resolution Astrophysics 2007 conference.

[2] Hanbury Brown,R., Twiss,R.Q, A test of a new type of stellar interferometer on Sirius, Nature 178, 1046-1048 (1956).

[3] Dravins, D., Barbieri, C., Da Deppo, V., et al. 2005, QuantEYE quantum optics instrumentation for astronomy. OWL Instrument Concept Study, Tech. rep., ESO, Document OWL-CSR-ESO-00000-0162

[4] ESO, OWL Concept Design Report, Tech. rep., 2005 ESO document OWL-TREESO-0000-0001 Issue 2

[5] G. Naletto, C. Barbieri, T. Occhipinti, I. Capraro et al,Iqueye, a single photon counting photometer applied to the ESO New Technology Telescope, Astronomy \& Astrophysics manuscript no. AA 200912862

[6] Mason, B., Gies, D., Hartkopf, W., et al. 1998, Astron. J., 115, 821

[7] Foellmi C. Intensity interferometry and the second-order correlation function g(2) in astrophysics A\&A Volume 507, Number 3, December I 2009 